\title{
Biofeedback no tratamento de transtornos relacionados ao estresse e à ansiedade: uma revisão crítica
}

\author{
Angélica da Silva Lantyer - Universidade Federal de São Paulo, Santos, Brasil \\ Milena de Barros Viana - Universidade Federal de São Paulo, Santos, Brasil \\ Ricardo da Costa Padovani - Universidade Federal de São Paulo, Santos, Brasil
}

\begin{abstract}
Resumo
O treinamento em biofeedback tem sido utilizado para o tratamento de diferentes quadros clínicos e para a prevenção/alívio de sintomas relacionados ao estresse/ansiedade. Este trabalho analisou a literatura de 2008 a 2012 sobre o tema "biofeedback, estresse e ansiedade" publicada nas bases MEDLINE, LILACS e Web of Sciences, utilizando como palavras-chave "biofeedback", "anxiety", "stress", "psychology" e "biofeedback training". Os resultados demonstram que técnicas de biofeedback são eficazes no manejo do estresse/ansiedade nas diferentes populações estudadas. Entretanto, todos os estudos encontrados foram realizados fora do Brasil, o que sugere que técnicas de biofeedback como ferramenta terapêutica não tem sido utilizadas no país, por algum motivo que merece ser melhor investigado.

Palavras-chave: Biofeedback, Psicologia, Ansiedade, Estresse, Transtornos de ansiedade.
\end{abstract}

Biofeedback in the treatment of stress and anxiety-related disorders: a critical review

\begin{abstract}
Biofeedback training has been utilized for the treatment of different pathological conditions, in particular those related to stress/anxiety. This study reviews the scientific literature from 2008 to 2012 about the subject "biofeedback, stress and anxiety", published in MEDLINE, LILACS and Web of Sciences, using as keywords "biofeedback", "anxiety", "stress", "psychology" and "biofeedback training". The results obtained showed that biofeedback training has been successfully employed as a therapeutic tool for the management of stress/anxiety in the different populations studied. Nevertheless, all of the studies found were performed outside Brazil, which suggests that biofeedback as a therapeutic tool has not been utilized in the country for some reason that deserves to be better investigated.

Keywords: Biofeedback, Psychology, Anxiety, Stress, Anxiety disorders.
\end{abstract}

Biofeedback en el tratamiento de trastornos relacionados con el estrés y la ansiedad: una revisión crítica

\begin{abstract}
Resumen
El entrenamiento con biofeedback se ha utilizado para el tratamiento de diferentes manifestaciones clínicas y para la prevención y alivio de síntomas relacionados con el estrés/ansiedad. Este estudio analizó la literatura desde 2008 hasta 2012 sobre el tema "biofeedback, estrés y ansiedad", publicada en MEDLINE, LILACS y Web of Sciences, utilizando como palabras clave "biofeedback", "anxiety", "stress", "psychology" y "biofeedback training". Los resultados demuestran que técnicas de biofeedback son eficaces para el tratamiento del estrés y la ansiedad en diferentes poblaciones. Sin embargo, todos los estudios encontrados se realizaron fuera de Brasil, un indicador de que el biofeedback como herramienta terapéutica no ha sido utilizado en el país por una razón que debe investigarse más a fondo.

Palabras clave: Biofeedback; Psicología, Ansiedad, Estrés, Trastornos de la ansiedad.
\end{abstract}

Técnicas de biofeedback proporcionam um retorno imediato de processos fisiológicos (frequência cardíaca, resposta galvânica da pele, tensão muscular, temperatura periférica, pressão arterial e atividade cerebral) dos quais o indivíduo pode não estar consciente ou apresentar dificuldades para controlar (Henriques, Keffer, Abrahamson \& Horst, 2011; McKee, 2008; Neves Neto, 2011, Strunk, Sutton \& Burns, 2009), permitindo o aprendizado da regulação voluntária de respostas fisiológicas e emocionais. O treinamento em biofeedback inclui diferentes métodos de conscientização e relaxamento, como técnicas musculares, respiratórias e cognitivas, que facilitam a autorregulação dos processos corporais (Cutshall \& cols., 2011; Elkins, Fisher \& Johnson, 2010; Mckee, 2008; Paul \& Garg, 2012).

Instrumentos de biofeedback podem também conter jogos. O participante vence quando alcança um Disponivel em www.scielo.br objetivo fisiológico, como manter a pressão arterial, por exemplo. Esses instrumentos, em virtude de seu caráter lúdico, têm sido inclusive utilizados com crianças (Jordanova \& Gucev, 2010; McKee, 2008).

Existem diferentes tipos de instrumentos de biofeedback. O biofeedback de variabilidade de frequência cardíaca (VFC), conhecido como HRV (Heart Rate Variability) biofeedback, por exemplo, é um dos mais confiáveis para a medição de parâmetros relativos ao funcionamento do sistema nervoso autônomo (Paul \& Garg, 2012). O biofeedback de VFC informa o índice de expressão emocional a partir da interação existente entre os sistemas simpático e parassimpático. Uma baixa VFC está associada a uma menor atividade do nervo vago e aumento da atividade do sistema nervoso simpático. Intervenções que aumentem a VFC e melhorem o tônus vagal podem diminuir a ansiedade. Um sensor de pulso preso nos dedos é conectado a um 
computador e mostra o ritmo cardíaco em tempo real em um gráfico. Um padrão gráfico denteado demonstra baixa coerência, indicando, portanto, níveis de estresse e ansiedade elevados. Um padrão gráfico com ondas suaves mostra alta coerência, bem-estar e menor índice de estresse. O biofeedback de VFC tem sido utilizado no tratamento de problemas cardiovasculares, transtorno de ansiedade generalizada, transtorno de pânico e transtorno do estresse póstraumático (TEPT) (Wheat \& Larkin, 2010).

Um outro instrumento de biofeedback, comumente utilizado em associação à VFC, é o RSA (Respiratory Sinus Arbythmia), que mensura a arritmia sinusal respiratória, sendo capaz de medir a variação da frequência respiratória. Tal equipamento tem como objetivo manter o ritmo respiratório em seis respirações por minuto, para que ocorra uma ressonância entre o ritmo respiratório e o baroreflexo (Zucker, Samuelson, Muench, Greenberg \& Gevirtz, 2009), aumentando a amplitude da VFC. Durante o relaxamento, o sistema nervoso parassimpático é ativado e as ondulações respiratórias e a taxa cardíaca são sincronizadas e aumentadas (Mikosch \& cols., 2010). Essas alterações são informadas em tempo real por meio de um sensor digital infravermelho.

Por sua vez o biofeedback eletromiográfico (EMG) mostra a atividade elétrica muscular em tempo real, buscando a própria regulação direta da atividade muscular com o objetivo de induzir o relaxamento (Nicholson, Buse, Andrasik, \& Lipton, 2011).

Já o biofeedback eletroencefalográfico (EEG) ou neurofeedback identifica os tipos de ondas cerebrais atuantes no momento, a partir de sensores instalados na cabeça do indivíduo. Ondas alfa têm sido associadas a relaxamento e bem-estar, enquanto ondas teta (produzidas com os olhos fechados) têm sido associadas a estados de meditação, início de sono ou hipnose (Bhat, 2010; Gruzelier, 2009). O treinamento alfa/teta (A/T) envolve a identificação das ondas alfa e teta durante o relaxamento do paciente, com os olhos fechados, frequentemente ao som de músicas tranquilas. O objetivo é aumentar a relação A/T.

$\mathrm{O}$ biofeedback de respostas galvânicas da pele (GSR) mede a resposta eletrodérmica mediante o posicionamento dos sensores nas pontas dos dedos indicador e médio da mão dominante do participante (Strunk \& cols., 2009). A condutividade nas extremidades é um importante indicador emocional.

Finalmente, o biofeedback termal também permite o acoplamento de sensores nas extremidades (mãos, dedos), sendo sensível à temperatura da pele por meio de pequenos vasos. Durante estados de maior relaxamento, os vasos dilatam e a temperatura aumenta
(Nestoriuc, Martin, Rief, \& Andrasik, 2008; Nicholson \& cols., 2011).

Embora técnicas de biofeedback sejam frequentemente utilizadas como instrumentos de medida da atividade do sistema nervoso autônomo e, consequentemente para aferir níveis de estresse/ansiedade, alguns estudos mais recentes tem se voltado para a sua utilização como ferramenta terapêutica. Evidências (Éismont, Lutsyuk, \& Pavlenko, 2011; Gervitz \& Dalenberg, 2008; Lande, Williams, Francis, Gragnani \& Morin, 2010) também sugerem que o emprego dessas técnicas como recurso terapêutico esteja sendo utilizado em particular para o manejo do estresse e da ansiedade. O objetivo deste trabalho foi analisar criticamente a literatura científica de 2008 a 2012 sobre o uso e a eficácia do biofeedback. como tratamento ou intervenção terapêutica nos transtornos de ansiedade ou em transtornos relacionados ao estresse e à ansiedade.

\section{Método}

As buscas pelos artigos foram feitas por três investigadores distintos nas bases de dados Medline, Lilacs e Web of Sciences. Foram utilizados os termos "biofeedback", "anxiety", "stress", "psychology" e "biofeedback training". Artigos relacionados ao tema de biofeedback e estresse/ansiedade recentes citados nas referências dos artigos selecionados também foram incluídos na análise.

Os critérios de inclusão foram: a língua (inglês, português ou espanhol), o ano de publicação (artigos publicados desde 2008; tendo em vista que foi em particular a partir dos últimos 5 anos que estudos realizados com uma das principais formas de biofeedback atualmente utilizada para o manejo do estresse/ansiedade, o biofeedback eletroencefalográfico, começam a ser publicados) (http://www.neurofeedback-scotland.com/anxietydisorders.html) e a utilização do biofeedback como abordagem terapêutica e não apenas como instrumento de medida. Foram excluídos artigos com: 1) metodologia não descrita em detalhes; 2) não-utilização da técnica para o diagnóstico/tratamento de transtornos mentais (definidos, segundo os Manual de diagnóstico e estatística dos transtornos mentais em sua 4a. versão revisada - DSM-IV-TR (APA, 2000), como uma alteração dos processos cognitivos e afetivos do desenvolvimento, perturbações de raciocínio, de comportamento, da compreensão da realidade ou de adaptação às condições da vida), ou, ainda, 3) estudos realizados com animais de laboratório. Também foram excluídos os artigos aos quais os pesquisadores não tiveram acesso em sua forma completa. 


\section{Resultados}

Dentre os 29 artigos analisados, três eram estudos de caso, 17 eram ensaios clínicos randomizados, um era um estudo no qual os grupos de comparação foram formados de forma não-aleatória (Tabela 1) e oito eram revisões de literatura (Tabela 2).

Tabela 1. Estudos que utilizaram técnicas de biofeedback com finalidade terapêutica

\begin{tabular}{|c|c|c|c|c|c|}
\hline Objetivos & Autores & País & População & Biofeedback & Eficácia \\
\hline \multirow{6}{*}{$\begin{array}{l}\text { Investigar a eficácia do } \\
\text { biofeedback na redução da } \\
\text { ansiedade }\end{array}$} & Bhat, 2010 & Índia & $\begin{array}{l}100 \text { pacientes } \\
\text { psiquiátricos }\end{array}$ & EEG & $\operatorname{Sim}$ \\
\hline & $\begin{array}{l}\text { Éismont \& } \\
\text { cols., } 2011\end{array}$ & Ucrânia & $\begin{array}{l}17 \text { crianças e } \\
\text { adolescentes }\end{array}$ & EEG & Sim \\
\hline & $\begin{array}{c}\text { Henriques \& } \\
\text { cols., } 2011\end{array}$ & EUA & $\begin{array}{l}44 \text { estudantes } \\
\text { universitários }\end{array}$ & VFC & Sim \\
\hline & $\begin{array}{l}\text { Mikosch \& } \\
\text { cols., } 2010\end{array}$ & Áustria & $\begin{array}{l}212 \text { pacientes } \\
\text { cardíacos }\end{array}$ & RSA & Sim \\
\hline & $\begin{array}{c}\text { Morarend \& } \\
\text { cols., } 2011\end{array}$ & EUA & $\begin{array}{l}81 \text { pacientes } \\
\text { odontológicos }\end{array}$ & RSA; VFC & Sim \\
\hline & $\begin{array}{c}\text { Paul \& Garg, } \\
2012\end{array}$ & Índia & $\begin{array}{l}30 \text { jogadores de } \\
\text { basquete }\end{array}$ & RSA; VFC & Sim \\
\hline \multirow{4}{*}{$\begin{array}{l}\text { Investigar a eficácia do } \\
\text { biofeedback na redução do } \\
\text { estresse }\end{array}$} & $\begin{array}{l}\text { Cutshall\& } \\
\text { cols., } 2011 \\
\end{array}$ & EUA & 8 enfermeiras & VFC & Sim \\
\hline & $\begin{array}{l}\text { Hallman \& } \\
\text { cols., } 2011\end{array}$ & Suécia & $\begin{array}{c}24 \text { pacientes com dor } \\
\text { crônica no pescoço ou } \\
\text { ombros } \\
\end{array}$ & VFC & Sim \\
\hline & $\begin{array}{l}\text { Jordanova \& } \\
\text { Gucev, } 2010\end{array}$ & Macedônia & $\begin{array}{c}30 \text { crianças saudáveis; } \\
30 \text { FC; } 30 \text { TAG; } 30 \\
\text { TDAH }\end{array}$ & VFC & Não \\
\hline & $\begin{array}{l}\text { Sherlin \& } \\
\text { cols., } 2010 \\
\end{array}$ & EUA & 43 adultos estressados & EEG; RSA & $\operatorname{Sim}$ \\
\hline \multirow{2}{*}{$\begin{array}{l}\text { Investigar a eficácia do } \\
\text { biofeedback para o manejo } \\
\text { do estresse/ ansiedade }\end{array}$} & $\begin{array}{c}\text { Yahav \& } \\
\text { Cohen, } 2008 \\
\end{array}$ & Israel & 255 adolescentes & GSR & Sim \\
\hline & Bradley, 2010 & EUA e NZ & $\begin{array}{l}136 \text { estudantes do } \\
\text { ensino médio }\end{array}$ & & Sim \\
\hline \multirow[t]{3}{*}{$\begin{array}{l}\text { Investigar a eficácia do } \\
\text { biofeedback } \\
\text { tratamento de TEPT }\end{array}$} & $\begin{array}{l}\text { Gervitz \& } \\
\text { Dalenberg, } \\
2008 \\
\end{array}$ & EUA & $\begin{array}{l}1 \text { adulto (estudo de } \\
\text { caso) }\end{array}$ & VFC & Sim \\
\hline & $\begin{array}{c}\text { Lande \& } \\
\text { cols., } 2010\end{array}$ & EUA & 39 Militares & VFC & Não \\
\hline & $\begin{array}{l}\text { Zucker \& } \\
\text { cols., } 2009\end{array}$ & EUA & $\begin{array}{l}38 \text { pacientes com } \\
\text { TEPT }\end{array}$ & RSA & Sim \\
\hline \multirow{3}{*}{$\begin{array}{l}\text { Investigar a eficácia do } \\
\text { biofeedback no tratamento } \\
\text { de quadros relacionados ao } \\
\text { estresse }\end{array}$} & $\begin{array}{l}\text { Kang \& cols., } \\
2009\end{array}$ & Coréia & $\begin{array}{l}32 \text { pacientes mulheres } \\
\text { com enxaqueca }\end{array}$ & Termal & $\operatorname{Sim}$ \\
\hline & $\begin{array}{l}\text { Slutsker \& } \\
\text { cols., } 2010\end{array}$ & Israel & $\begin{array}{c}\text { (estudo de caso) } \\
\text { adolescente com } \\
\text { síndrome do vômito } \\
\text { cíclico }\end{array}$ & VFC & $\operatorname{Sim}$ \\
\hline & $\begin{array}{l}\text { McLay \& } \\
\text { Spira, } 2009\end{array}$ & EUA & $\begin{array}{c}\text { (estudo de caso) militar } \\
\text { com insônia }\end{array}$ & VFC & Sim \\
\hline
\end{tabular}


Tabela 1. Estudos que utilizaram técnicas de biofeedback com finalidade terapêutica (continuação)

\begin{tabular}{|c|c|c|c|c|c|c|}
\hline Objetivos & & Autores & País & População & Biofeedback & Eficácia \\
\hline \multirow[t]{3}{*}{$\begin{array}{l}\text { Biofeedback verdadeiro } \\
\text { falso }\end{array}$} & $\mathrm{x}$ & $\begin{array}{l}\text { Lazarov \& } \\
\text { cols., } 2010\end{array}$ & Israel & $\begin{array}{c}80 \text { universitários com } \\
\text { TOC }\end{array}$ & GSR & $\begin{array}{l}\text { Falso } \\
\text { BFB }\end{array}$ \\
\hline & & $\begin{array}{l}\text { Lazarov \& } \\
\text { cols., } 2012\end{array}$ & Israel & $\begin{array}{c}76 \text { universitários com } \\
\text { TOC }\end{array}$ & EMG, GSR. & $\begin{array}{l}\text { Falso } \\
\text { BFB }\end{array}$ \\
\hline & & $\begin{array}{l}\text { Strunk \& } \\
\text { cols., } 2009\end{array}$ & EUA & 63 universitários & $\begin{array}{l}\text { EMG, GSR, } \\
\text { Termal, VFC }\end{array}$ & Não \\
\hline
\end{tabular}

EUA: Estados Unidos da América; EMG: eletromiograma; EEG: eletroencefalograma; GSR: respostas galvânicas da pele; RSA: arritmia sinusal respiratória; VFC: variabilidade da frequência cardíaca; NZ: Nova Zelândia; FC: fibrose cística; TAG: transtorno de ansiedade generalizada; TDAH: transtorno do déficit de atenção; TEPT: transtorno do estresse pós-traumático.

Dezesseis estudos utilizaram o biofeedback de VFC (Bradley \& cols., 2010; Cutshall \& cols., 2011; Gervitz \& Dalenberg, 2008; Gruzelier, 2009; Hallman, Olsson, Schéele, Melin \& Lyskov, 2011; Henriques \& cols., 2011; Jordanova \& Gucev, 2010; Lande \& cols., 2010; McGrady, 2010; McLay \& Spira, 2009; Morarend, Spector, Dawson, Clark \& Holmes, 2011; Paul \& Garg, 2012; Shenefelt, 2010; Slutsker, Konichezkya \& Gothelf, 2010; Strunk \& cols., 2009; Wheat \& Larkin, 2010) e o RSA (McGrady, 2010; Mikosch \& cols., 2010; Morarend, Spector, Dawson, Clark \& Holmes, 2011; Sherlin, Muench \& Wyckoff, 2010; Zucker \& cols., 2009) foram mencionados em cinco estudos. Já o biofeedback termal (Kang, Park, Chung \& Yu, 2009; Nestoriuc \& cols., 2008; Nicholson \& cols., 2011;
Strunk \& cols., 2009) o EEG (Éismont \& cols., 2011; Gruzelier, 2009; Nestoriuc \& cols., 2008; Sherlin \& cols., 2010) e o EMG (Lazarov \& cols., 2012; Nestoriuc \& cols., 2008; Nicholson \& cols., 2011; Strunk \& cols., 2009) foram mencionados em quatro estudos. Os estudos consultados foram realizados em diferentes países, como Estados Unidos (59\%), Israel $(14 \%)$, Índia (7\%), Inglaterra (3\%), Áustria (3\%), Coreia (3\%), Suécia (3\%), Ucrânia $(3 \%)$ e Macedônia (3\%), o que sugere que a eficácia das técnicas de biofeedback não se encontra sob a influência de fatores culturais. Não foram encontrados na literatura estudos brasileiros que fizessem uso de técnicas de biofeedback como ferramenta terapêutica para o manejo do estresse/ansiedade.

Tabela 2. Revisões de estudos que utilizaram técnicas de biofeedback com finalidade terapêutica

\begin{tabular}{|c|c|c|c|c|}
\hline Autores & País & Assunto & $\begin{array}{l}\text { Tipos de } \\
\text { biofeedback }\end{array}$ & Resultados \\
\hline
\end{tabular}

Nestoriuc \& $\quad$ EUA e Biofeedback para o Termal, EMG, O treinamento em biofeedback
cols., 2008 Alemanha tratamento de cefaleias. EEG, GSR reduziu sintomas de ansiedade e depressão. O EMG mostrou redução na tensão nas áreas relacionadas à dor.

\begin{tabular}{|c|c|c|c|c|}
\hline $\begin{array}{ll}\text { Meuret } & \& \\
\text { Ritz, 2010 } & \end{array}$ & EUA & $\begin{array}{l}\text { Biofeedback no tratamento } \\
\text { da asma e transtorno de } \\
\text { pânico. }\end{array}$ & & $\begin{array}{l}\text { Os estudos foram inconclusivos em } \\
\text { ambos os casos (transtorno do } \\
\text { pânico e asma). }\end{array}$ \\
\hline $\begin{array}{l}\text { McGrady, } \\
2010\end{array}$ & EUA & $\begin{array}{lll}\text { Biofeedback para } & \text { o } \\
\text { tratamento da } & \text { diabetes } & \text { e } \\
\text { hipertensão } & & \end{array}$ & RSA; VFC & $\begin{array}{l}\text { Estudo concluiu que o biofeedback } \\
\text { não tem a mesma eficácia para todos } \\
\text { os pacientes. }\end{array}$ \\
\hline $\begin{array}{l}\text { Nicholson } \& \\
\text { cols., } 2011\end{array}$ & EUA & $\begin{array}{l}\text { Biofeedback para o } \\
\text { tratamento de enxaqueca } \\
\text { e cefaleia tensional. }\end{array}$ & $\begin{array}{c}\text { Termal e } \\
\text { EMG }\end{array}$ & $\begin{array}{l}47 \text { a } 70 \% \text { dos tratados com } \\
\text { biofeedback apresentaram redução das } \\
\text { dores de cabeça, e melhora dos } \\
\text { sintomas depressivos e de ansiedade. }\end{array}$ \\
\hline $\begin{array}{l}\text { Shenefelt, } \\
2010\end{array}$ & EUA & $\begin{array}{l}\text { Biofeedback para } \mathrm{O} \\
\text { tratamento de doenças de } \\
\text { pele relacionadas ao } \\
\text { estresse. }\end{array}$ & VFC, GSR & $\begin{array}{l}\text { O treinamento em biofeedback } \\
\text { reduziu o estresse (desencadeador de } \\
\text { problemas dermatológicos), } \\
\text { mostrando eficaz. }\end{array}$ \\
\hline
\end{tabular}


Tabela 2. Revisões de estudos que utilizaram técnicas de biofeedback com finalidade terapêutica

\begin{tabular}{|c|c|c|c|c|}
\hline Autores & País & Assunto & $\begin{array}{l}\text { Tipos de } \\
\text { biofeedback }\end{array}$ & Resultados \\
\hline $\begin{array}{l}\text { Wheat \& } \\
\text { Larkin, } 2010\end{array}$ & EUA & $\begin{array}{l}\text { Mediu a eficácia do } \\
\text { biofeedback de VFC e de } \\
\text { outros equipamentos de } \\
\text { biofeedback relacionados } \\
\text { com a melhora da VFC e } \\
\text { baroreflexo. }\end{array}$ & VFC & $\begin{array}{l}\text { O HRV melhora a VFC, além de } \\
\text { trazer alívio de sintomas clínicos. } \\
\text { Nem sempre os resultados clínicos } \\
\text { concordam com os fisiológicos. Não } \\
\text { foi estabelecida relação entre a } \\
\text { duração da prática de biofeedback e } \\
\text { sua eficácia. }\end{array}$ \\
\hline $\begin{array}{l}\text { Elkins \& } \\
\text { cols., } 2010\end{array}$ & EUA & $\begin{array}{l}\text { Investigou a eficácia de } \\
\text { equipamentos de } \\
\text { biofeedback e de outras } \\
\text { práticas terapêuticas para } \\
\text { o alívio de sintomas de } \\
\text { pacientes com câncer. }\end{array}$ & & $\begin{array}{l}\text { Biofeedback associado a relaxamento } \\
\text { mostrou-se eficaz na redução da dor } \\
\text { de pacientes com câncer em } \\
\text { comparação com a técnica de } \\
\text { atenção concentrada. }\end{array}$ \\
\hline $\begin{array}{l}\text { Gruzelier, } \\
2009\end{array}$ & Inglaterra & $\begin{array}{l}\text { Biofeedback para melhora } \\
\text { da relação das ondas } \\
\text { teta/alfa e indução de } \\
\text { relaxamento. }\end{array}$ & EEG; VFC & $\begin{array}{l}\text { O treinamento A/T com } \\
\text { relaxamento e música mostrou } \\
\text { melhora no bem-estar de estudantes, } \\
\text { músicos e melhora no desempenho } \\
\text { de músicos e dançarinos nos estudos } \\
\text { revisados. }\end{array}$ \\
\hline
\end{tabular}

EUA: Estados Unidos da América; EMG: eletromiográfico; EEG: eletroencefalográfico; GSR: respostas galvânicas da pele; RSA: arritmia sinusal respiratória; VFC: variabilidade da frequência cardíaca. A/T: ondas alfa/ondas teta

\section{Discussão}

Os artigos consultados demonstraram que a aplicação de técnicas de biofeedback tem sido realizada para o manejo do estresse e da ansiedade resultantes de diferentes situações. Por exemplo, técnicas de biofeedback foram utilizadas no manejo do estresse diário em enfermeiras (Cutshall \& cols., 2011), para melhorar o desempenho e diminuir a ansiedade de jogadores de basquete (Paul \& Garg, 2012), músicos (Gruzelier, 2009) ou estudantes do ensino médio ou universitário (Bradley \& cols., 2010; Yahav \& Cohen, 2008). Ademais, essas técnicas também parecem ter sido úteis para o alívio de sintomas oriundos de diferentes quadros clínicos, como por exemplo, dores crônicas (Hallman \& cols., 2011; Kang \& cols., 2009; Nestoriuc \& cols., 2008; Nicholson \& cols., 2011), asma (Meuret \& Ritz, 2010), doenças cardiovasculares (McGrady, 2010; Mikosch \& cols., 2010), doenças de pele relacionadas ao estresse (Shenefelt, 2010), transtorno de pânico (Meuret \& Ritz, 2010) e outros transtornos ansiosos (Jordanova \& Gucev, 2010; Sherlin \& cols., 2010; Slutsker \& cols., 2010).

Os três estudos de casos analisados apontaram para alguns aspectos inovadores da utilização de técnicas de biofeedback (Gervitz \& Dalenberg, 2008; McLay \& Spira, 2009; Slutsker \& cols., 2010). Um dos estudos de caso fez uso de técnicas de biofeedback para o tratamento de um quadro de insônia (McLay \& Spira,
2009). O paciente, um médico das forças armadas americanas em missão no Iraque, recebeu um dispositivo portátil de biofeedback de VFC (da marca Stress Eraser). Embora esse paciente já houvesse passado por seis sessões de terapia cognitivocomportamental (TCC) antes da introdução da técnica, a opção pelo uso do biofeedback se deu em razão da dificuldade de acompanhamento psicológico frequente em um ambiente de guerra. O paciente foi orientado a utilizar o dispositivo antes de dormir. Apresentou melhora do padrão de sono, que se manteve mesmo depois do follow-up, realizado após uma e seis semanas. Após um ano, o paciente continuava a não apresentar dificuldades para dormir. Desenvolveu, entretanto, um quadro de TEPT, o que demonstra que, embora o biofeedback tenha sido eficaz para o tratamento da insônia, não foi capaz de prevenir o surgimento de outros transtornos.

O segundo caso clínico estudado utilizou técnicas de biofeedback para o tratamento de um quadro de ansiedade (Slutsker \& cols., 2010). O paciente, um garoto de 13 anos diagnosticado com transtorno de ansiedade generalizada, que apresentava como sintoma comórbido vômitos, recebeu o mesmo dispositivo de biofeedback descrito acima. Após 16 sessões de TCC, que envolvia psicoeducação, identificação dos disparadores do vômito, reestruturação cognitiva e treinamento em biofeedback, o paciente apresentou 
redução significativa dos sintomas (Slutsker \& cols., 2010).

No terceiro estudo, o biofeedback de VFC também foi utilizado juntamente com sessões de TCC, focadas no tratamento de um quadro de TEPT. Os resultados mostraram uma redução da pontuação do paciente de 75 para 50 no Inventário de Sintomas de Trauma (Briére, 1995), um questionário de 100 itens em que uma maior pontuação indica a maior presença de sintomas traumáticos. Além disso, passados três meses do início da intervenção, o paciente apresentou remissão do sintoma de agorafobia (Gervitz \& Dalenberg, 2008).

Outros estudos consultados também investigaram os efeitos da utilização de técnicas de biofeedback no tratamento de quadros de TEPT (Lande \& cols., 2010; Zucker \& cols., 2009). Um deles comparou o RSA com o relaxamento muscular progressivo (RMP), como intervenções complementares para 38 pessoas com sintomas de TEPT em uma instalação de tratamento residencial para transtorno por uso de substâncias (Zucker \& cols., 2009). O outro, visando estudar a eficácia do biofeedback como tratamento complementar de TEPT em 49 soldados em combate, demonstrou que tanto o grupo experimental quanto o grupo controle apresentaram diminuição dos sintomas de ansiedade e depressão após a intervenção, não sendo possível visualizar o efeito específico do biofeedback (Lande \& cols., 2010).

Alguns estudos demonstraram que o biofeedback é um tratamento eficaz para dores de cabeça crônicas ou enxaquecas (Kang \& cols., 2009; Nestoriuc \& cols., 2008; Nicholson \& cols., 2011). Duas revisões sobre cefaleia tensional e enxaqueca mostraram que cerca de 47 a $70 \%$ dos pacientes tratados com técnicas de biofeedback apresentaram uma redução significativa na frequência das dores de cabeça, além de demonstrarem melhora em sintomas depressivos e ansiosos (Nicholson \& cols., 2011). Um ensaio clínico coreano realizado em mulheres com enxaqueca demonstrou eficácia da utilização do biofeedback termal (Procomp Biofeedback 2.1). Adicionalmente, esse estudo mostrou que pacientes com enxaqueca possuem níveis mais altos de ansiedade e depressão que a população saudável. Com a utilização do biofeedback, houve redução também desses sintomas (Kang \& cols., 2009). Outros estudos também apresentaram bons resultados com a utilização do EMG, EEG e GSR (Nestoriuc \& cols., 2008; Nicholson \& cols., 2011), para o tratamento dos mesmos quadros clínicos.

Adultos com problemas cardiovasculares, como os submetidos à angiografia coronariana e aqueles com diabetes e hipertensão, também foram submetidos a sessões de biofeedback (McGrady, 2010; Mikosch \& cols., 2010). Os resultados obtidos sugerem que, além de atuar na melhora dos níveis de ansiedade, a utilização de técnicas de biofeedback também auxilia o controle da pressão arterial e frequência cardíaca. Nesses casos, o RSA mostrava em tempo real o ritmo influenciado pelo efeito do baroreflexo e o ritmo da arritmia sinusal respiratória, que modulam a atividade autonômica. Sendo assim, os pacientes podiam perceber as alterações em sua respiração e taxas cardíacas, que foram aprendidas a partir da técnica da respiração abdominal (McGrady, 2010; Mikosch \& cols., 2010). Entretanto, o estudo indica também a importância de se investigar por que alguns pacientes respondem bem ao tratamento com biofeedback, enquanto outros têm dificuldades para aprender a controlar suas respostas fisiológicas.

Nesse sentido, é importante também mencionar um estudo de revisão realizado nos Estados Unidos, cujo objetivo foi investigar a eficácia do biofeedback de VFC e de outros equipamentos de biofeedback relacionados com a melhora da VFC e baroreflexo. Os autores concluíram que o biofeedback de VFC melhora a VFC, além de diminuir a incidência de alguns dos sintomas clínicos dos pacientes. Entretanto, o mecanismo pelo qual essas alterações se dão não é claro, pois nem sempre os resultados clínicos concordam com as medidas fisiológicas. Também não foi encontrada relação entre a duração da prática de biofeedback e sua eficácia.

O manejo da ansiedade e do estresse traz benefícios não apenas para pessoas portadoras de quadros patológicos, mas também melhora o desempenho de indivíduos expostos frequentemente a situações que geram ansiedade. É o caso, por exemplo, de atletas e músicos. A ansiedade que precede apresentações ou campeonatos é descrita por esses sujeitos como alta, podendo acarretar comprometimento do desempenho do indivíduo. Em um estudo com trinta jogadores de basquete (Paul \& Garg, 2012) com idade entre 18 e 28 anos, o biofeedback de VFC reduziu a ansiedade antes dos jogos e melhorou o desempenho dos atletas, que foi medido a partir dos itens: passe, arremesso e drible da bola. Foram utilizadas técnicas de manejo de estresse como RMP, respiração profunda e visualização, durante 10 dias consecutivos associados ao biofeedback (Biograph Procomp Infinity 5.0), por 20 minutos diários.

No caso dos músicos, um biofeedback eletroencefalográfico (EEG) foi utilizado para medir padrões de atividade neuronal, com o objetivo de estabelecer um estado de relaxamento a partir do aumento da razão alfa/teta (A/T). Isso foi realizado com base em informações auditivas. $\mathrm{O}$ estudo demonstrou que o EEG diminuiu a ansiedade pré- 
apresentações e melhorou o desempenho musical, além de diminuir o estresse percebido (Gruzelier, 2009).

Em uma pesquisa realizada com enfermeiras de um hospital norte-americano, que apresentavam elevados níveis de estresse no trabalho, equipamentos de biofeedback de VFC foram distribuídos e os sujeitos foram instruídos a fazerem uso desses equipamentos em casa, durante 30 minutos por dia, quatro vezes por semana, por um período total de quatro semanas (Cutshall \& cols., 2011). Os resultados indicaram que esse programa de biofeedback (Wild Divine) foi eficaz na redução do estresse dessas profissionais. Os resultados obtidos corroboraram observações realizadas em anos anteriores, e que sugerem a eficácia da utilização de técnicas de biofeedback na redução do estresse em diferentes profissionais de saúde (Oman, Hedbelg \& Thoresen, 2006), bem como em estudantes de medicina (Paul, Elam \& Verhulst, 2007).

Outro estudo norte-americano fez uso do RSA e do EEG em 43 participantes com níveis elevados de estresse e demonstrou, a partir da aplicação do teste de Stroop como estressor, que o RSA induziu um aumento de ondas alfa e uma diminuição de ondas beta, em áreas críticas para a regulação do estresse, o que se correlaciona positivamente com a diminuição do estresse (Sherlin \& cols., 2010).

Entre as quatro pesquisas com estudantes universitários analisadas (Henriques \& cols., 2011; Lazarov \& cols., 2010, 2012; Strunk \& cols., 2009), uma delas relatou melhora na ansiedade após 20 sessões de HRV (Henriques \& cols., 2011). A outra trouxe uma comparação entre biofeedback correto e falso, não tendo demonstrado diferença significativa entre ambos no manejo do estresse entre estudantes, muito embora tenha indicado mudanças em parâmetros relacionados ao relaxamento físico e mental (Strunk \& cols., 2009). Essa observação sugere que o estresse percebido é uma questão de difícil investigação e aponta para a necessidade de realização de estudos adicionais para investigar o que de fato mudaria a autopercepção do estresse. Já os outros dois estudos utilizaram o falso biofeedback de GSR para compreender as tendências obsessivo-compulsivas apresentadas pelo grupo de pacientes estudado (Lazarov \& cols., 2010; 2012). Os resultados observados demonstraram que os pacientes obsessivo-compulsivos confiavam mais no equipamento de biofeedback na hora de julgar a própria tensão muscular, mesmo quando a informação era falsa. Os autores interpretam esse resultado argumentando que, possivelmente por apresentarem dificuldades na auto-percepção dos sentimentos, os pacientes obsessivo-compulsivos procurariam informações externas ou regras, substitutas das informações corporais.
Em outro estudo analisado, 135 estudantes do ensino médio foram sujeitos de uma intervenção cognitivo-comportamental, associada a um equipamento de biofeedback de VFC. A intervenção tinha o objetivo de ensinar aos alunos técnicas de manejo de emoções, capacitando-os fundamentalmente a lidar com o estresse e a ansiedade. Foram observadas melhora na VFC dos estudantes que receberam a intervenção e diminuição significativa de parâmetros relacionados à ansiedade e ao estresse pré-avaliações (Bradley \& cols., 2010).

Entre os 255 estudantes adolescentes israelenses pesquisados, o biofeedback termal, também acompanhado de uma intervenção cognitivo comportamental, mostrou uma redução significativa na ansiedade pré-avaliações (Yahav \& Cohen, 2008). Por outro lado, um estudo comparativo, realizado na Macedônia com crianças saudáveis, crianças portadoras de transtorno de ansiedade generalizada (TAG), transtorno do déficit de atenção e hiperatividade (TDAH), e fibrose cística (FC) não apontou para diferenças significativas na redução da ansiedade e no desempenho dos jogos que compunham o biofeedback. (que eram pontuados). Cabe mencionar, com relação a este estudo, entretanto, que a utilização do equipamento foi realizada em uma única sessão. É possível, portanto, que uma intervenção mais prolongada pudesse trazer resultados diferentes (Jordanova \& Gucev, 2010). Na Ucrânia, 17 crianças saudáveis de 10 a 14 anos foram submetidas a um treinamento em biofeedback eletroencefalográfico (EEG) mediante sinais visuais ou auditivos. Após 12 sessões de 30 minutos, realizadas duas vezes por semana, foi observado um aumento na amplitude do ritmo alfa em quase todos os indivíduos testados, acompanhado por um aumento da relação $\mathrm{A} / \mathrm{T}$ em diferentes regiões encefálicas. De acordo com os autores, a maior incidência de ritmos alfa demonstrado no EEG significa aumento da eficácia da transmissão sináptica nas redes neurais no tálamo, sistema límbico e neocórtex, que são ativadas pelo sistema dopaminérgico (Éismont \& cols., 2011). As mudanças nos padrões de ondas cerebrais, acompanhadas pela diminuição da ansiedade no grupo experimental, sugerem que este tipo de biofeedback é eficaz para o manejo da ansiedade em crianças e adolescentes.

\section{Conclusões}

A maioria dos artigos consultados demonstrou eficácia da utilização de equipamentos de biofeedback como recurso terapêutico no manejo da ansiedade e do estresse, e de transtornos relacionados (insônia, asma, TP, TEPT, síndrome do vômito cíclico) (Hallman \& 
cols., 2011; Kang \& cols., 2009; Meuret \& Ritz, 2010; Nestoriuc \& cols., 2008; Nicholson \& cols., 2011; Shenefelt, 2010; Slutsker \& cols., 2010). Apenas quatro dos artigos pesquisados demonstraram que as técnicas de biofeedback utilizadas não apresentaram eficácia sobre outras abordagens terapêuticas ou não foram eficazes em reduzir de maneira significativa os sintomas almejados (Jordanova \& Gucev, 2010; Lande \& cols., 2010; Meuret \& Ritz, 2010; Nestoriuc \& cols., 2008).

Embora deva ser levada em consideração a possibilidade de que alguns estudos que apresentaram resultados negativos não tenham sido publicados, o grande número de resultados positivos divulgados sugere que técnicas de biofeedback podem ser úteis como ferramentas terapêuticas para o manejo do estresse/ansiedade. Nesse sentido, é importante também salientar que, embora a literatura estrangeira sobre o assunto seja vasta, nenhum estudo publicado no Brasil foi encontrado. Esta observação sugere que a utilização de técnicas de biofeedback como ferramenta terapêutica para o manejo do estresse/ansiedade no país esteja sendo, por alguma razão que merece ser mais bem explorada, negligenciada.

Com relação a essa última observação, algumas possibilidades podem ser levantadas. Por exemplo, equipamentos de biofeedback para o manejo do estresse/ansiedade não são produzidos no país. Necessitam ser importados, são caros, e os softwares encontram-se, em geral, na língua inglesa, o que dificulta também sua utilização. Uma alternativa possível para essa última dificuldade mencionada seria a tradução do material pelo próprio grupo de pesquisa, seguida pela validação posterior do instrumento em sua versão traduzida.

\section{Referências}

APA - American Psychiatric Association (2000). Manual diagnóstico e estatístico de transtornos mentais (DSM - IV-TR) (4a ed.). Porto Alegre/RS: Artes Médicas.

Bhat, P. (2010). Efficacy of Alfa EEG wave biofeedback in the management of anxiety. Industrial Psychiatry Journal, 19(2), 111-114.

Briére, J. (1995). Manual for the Trauma Symptom Inventory. Odessa, FL: Psychological Assessment Resources

Bradley, R.T., McCraty, R., Atkinson, M., Tomasino, D., Daugherty, A \& Arguelles, L. (2010). Emotion self-regulation, psychophysiological coherence, and test anxiety: results from an experiment using electrophysiological measures. Applied Psychophysiology and Biofeedback, 35, 261-283.
Cutshall, S. M., Wentworth, L. J., Wahner-Roedler, D. L., Vincent, A., Schmidt, J.E., Loehrer, L. L., Cha, S. S. \& Bauer, B. A. (2011). Evaluation of a biofeedback-assisted meditation program as a stress management tool for hospital nurses: a pilot study. Explore, 7(2), 110-112.

Éismont, E. V., Lutsyuk, N. V. \& Pavlenko, V. B. (2011). Moderation of increased anxiety in children and teenagers with the use of neurotherapy: estimation of the efficacy. Neirofiziologiya/Neurophysiology, 43(1), 63-72.

Elkins, G., Fisher, W. \& Johnson, A. (2010). Mindbody therapies in integrative oncology. Current Treatment Options in Oncology, 11, 128-140.

Gervitz, R. N. \& Dalenberg, C. (2008). Heart rate variability biofeedback in the treatment of trauma symptoms. Biofeedback, 36(1), 22-23.

Gruzelier, J. (2009). A theory of alpha/theta neurofeedback, creative performance enhancement, long distance functional connectivity and psychological integration. Cognitive Processing, 10, 101-109.

Hallman, D. M., Olsson, E. M., Schéele, B., Melin, L. \& Lyskov, E. (2011). Effects of Heart rate variability biofeedback in subjects with stressrelated chronic neck pain: a pilot study. Applied Psychophysiology and Biofeedback, 36, 71-80.

Henriques, G., Keffer, S., Abrahamson, C. \& Horst, S.J. (2011). Exploring the effectiveness of a computer-based heart rate variability biofeedback program in reducing anxiety in college students. Applied Psychophysiology and Biofeedback, 36,101-112.

Jordanova, N. P. \& Gucev, Z. (2010). Game-based peripheral biofeedback for stress assessment in children. Pediatrics International, 52, 428-431.

Kang, E. H., Park, J. E., Chung, C. S. \& Yu, B. H. (2009). Effect of biofeedback-assisted autogenic training on headache activity and mood states in korean female migraine patients. Journal of Korean Medical Science, 24, 936-940.

Lande, R. G., Williams, L. B., Francis, J. L., Gragnani, C. \& Morin, M. L. (2010). Efficacy of biofeedback for post-traumatic stress disorder. Complementary Therapies in Medicine, 18, 256-259.

Lazarov, A., Dar, R., Liberman, N. \& Oded, Y. (2012). Obsessive-compulsive tendencies and undermined confidence are related to reliance on proxies for internal states in a false feedback paradigm. Journal of Behavioral Therapy \& Experimental Psychiatry, 43, 556-564.

Lazarov, A., Dar, R., Liberman, N. \& Oded, Y. (2010). Are obsessive-compulsive tendencies related to reliance on external proxies for internal states? 
Evidence from biofeedback-aided relaxation studies. Behaviour Research and Therapy, 48, 516-523.

McGrady, A. (2010) The effects of biofeedback in diabetes and essential hypertension. Cleveland Clinic Journal of Medicine, 77(3).

McKee, M. G. (2008) Biofeedback: an overview in the context of heart-brain medicine. Cleveland Clinic Journal of Medicine, 75(2).

McLay, R. N. \& Spira, J. L. (2009) Use of a portable biofeedback device to improve insomnia in a combat zone, a case Report. Applied Psychophysiology and Biofeedback, 34, 319-321.

Meuret, A. E. \& Ritz, T. (2010). Hyperventilation in panic disorder and asthma: empirical evidence and clinical strategies. International Journal of Psychophysiology, 78, 68-79.

Mikosch, P., Hadrawa, T., Laubreiter, K., Brandl, J., Pilz, J., Stettner, H. \& Grimm, G. (2010). Effectiveness of respiratory-sinus-arrhythmia biofeedback on state-anxiety in patients undergoing coronary angiography. Journal of Advanced Nursing, 66(5), 1101-1110.

Morarend, Q. A., Spector, M. L., Dawson, D. V., Clark, S. H. \& Holmes, D. C. (2011). The use of a respiratory rate biofeedback device to reduce dental anxiety: an exploratory investigation. Applied Psychophysiology and Biofeedback, 36, 63-70.

Nestoriuc, Y., Martin, A., Rief, W. \& Andrasik, F. (2008). Biofeedback treatment for headache disorders: a comprehensive efficacy review. Applied Psychophysiology and Biofeedback, 33, 125-140.

Neves Neto, A. R. (2011) Técnicas de respiração para a redução do estresse em terapia cognitivocomportamental. Arquivos Médicos dos Hospitais e da Faculdade de Ciências Médicas da Santa Casa, 56(3), 158-68.

Nicholson, R. A., Buse, D. C., Andrasik, F. \& Lipton, R.B. (2011). Nonpharmacologic treatments for migraine and tension-type headache: how to choose and when to use. Current Treatment Options in Neurology, 13, 28-40.

Oman, D., Hedberg J. \& Thoresen, C.E. (2006). Passage meditation reduces perceived stress in health professionals: a randomized, controlled trial. Journal of Consulting and Clinical Psychology, 74, 714-719.
Paul, G., Elam, B. \& Verhulst, S. J. (2007). A longitudinal study of students' perceptions of using deep breathing meditation to reduce testing stresses. Teaching and Learning in Medicine, 19, 287292.

Paul, M. \& Garg, K. (2012). The effect of heart rate variability biofeedback on performance psychology of basketball players. Applied Psychophysiology and Biofeedback, 37, 131-144.

Shenefelt, P.D. (2010). Psychological interventions in the management of common skin conditions. Psychology Research and Behavior Management, 3, 51-63.

Sherlin, L., Muench, F. \& Wyckoff, S. (2010). Respiratory sinus arrhythmia feedback in a stressed population exposed to a brief stressor demonstrated by quantitative EEG and sLORETA. Applied Psychophysiology and Biofeedback, 35, 219-228.

Slutsker, B., Konichezkya, A. \& Gothelf, D. (2010). Breaking the cycle: cognitive behavioral therapy and biofeedback training in a case of cyclic vomiting syndrome. Psychology, Health \& Medicine, 15(6), 625-631.

Strunk, K. K, Sutton, G. W. \& Burns. N. S. (2009). Beneficial effects of accurate and false brief. Biofeedback on Relaxation, Perceptual and Motor Skills, 109(3), 881-886.

Yahav, R. \& Cohen, M. (2008). Evaluation of a cognitive-behavioral intervention for adolescents. International Journal of Stress Management, 15(2), 173188.

Wheat, A. L. \& Larkin K. T. (2010). Biofeedback of heart rate variability and related physiology: a critical review. Applied Psychophysiology and Biofeedback, 35, 229-242.

Zucker, T.L., Samuelson, K. W., Muench, F., Greenberg, M. A. \& Gevirtz, R. N. (2009). The effects of respiratory sinus arrhythmia biofeedback on heart rate variability and posttraumatic stress disorder symptoms: a pilot study. Applied Psychophysiology and Biofeedback, 34(2), 135-43.

Recebido em: 03/09/2012

Reformulado em: 26/02/2013

Aprovado em: 14/03/2013 
Sobre os autores:

Angélica da Silva Lantyer é graduada em Psicologia e mestranda no Programa de Mestrado Interdisciplinar em Ciências da Saúde, ambos pela Universidade Federal de São Paulo, com atuação na área de ansiedade, estresse e biofeedback. É bolsista CAPES.

Milena de Barros Viana é graduada em Psicologia pela Universidade Federal de São Paulo, com doutorado em Psicobiologia, pela Universidade de São Paulo, e em Epistemologia da Psicologia, pela Universidade Federal de São Carlos. Atualmente, é professora adjunta da Universidade Federal de São Paulo, atuando na área da neurobiologia da ansiedade.

Ricardo da Costa Padovani é psicólogo, doutor em Educação Especial pela Universidade Federal de São Carlos, especialista em Terapia Comportamental Cognitiva em Saúde Mental pelo Instituto de Psiquiatria HCFMUSP/Programa de Ansiedade e professor adjunto II do curso de Psicologia da Universidade Federal de São Paulo - Campus Baixada Santista.

Contato com os autores:

Departamento de Biociências da UNIFESP - Campus Baixada Santista

Av. D. Ana Costa, 95 - Vl. Mathias - CEP 11060-001 - Santos/SP, Brasil.

E-mail: milenabv@gmail.com

Houve a correção na ordem dos autores.

Publicado:

Ricardo da Costa Padovani - Universidade Federal de São Paulo, Santos, Brasil

Milena de Barros Viana- Universidade Federal de São Paulo, Santos, Brasil

Angélica da Silva Lantyer - Universidade Federal de São Paulo, Santos, Brasil

Correto:

Angélica da Silva Lantyer - Universidade Federal de São Paulo, Santos, Brasil

Milena de Barros Viana- Universidade Federal de São Paulo, Santos, Brasil

Ricardo da Costa Padovani - Universidade Federal de São Paulo, Santos, Brasil 